\title{
Quantum Fluctuations of Low Dimensional Bose-Einstein Condensates in Optical Systems
}

\author{
Alem Mebrahtu \\ Department of Physics, College of Natural and Computational Sciences, P.O. Box 3044, Mekelle \\ University, Mekelle, Tigray, Ethiopia (Alem.mebrahtu@mu.edu.et)
}

\begin{abstract}
A system of low dimensional condensed ultracold atomic gases inside a field of a laser-driven optical cavity exhibits dispersive optical bistability. During such a process the system also shows quantum fluctuations. Condensate fluctuations are highly manifested particularly in low dimensional systems. In this paper we have investigated the theory and manifestation of fluctuations in quantum optical systems in low dimensional Bose-Einstein condensates. We have described the system using the mean-field approximation. In this study we have verified that low dimensional quantum gases exhibit not only highly fascinating properties but also we have indicated that the use of mean field theory to describe quantum gases in low dimensions is highly restricted since the possibility of generating low dimensional bosonic condensates is dominated by the existence of highly sensitive and intrinsic quantum fluctuations.
\end{abstract}

Keywords: Bose-Einstein condensates, Optical bistability, Quantum fluctuations, Optomechanics, Low dimensionality, Radiation field

\section{INTRODUCTION}

Quantum fluctuations are variations or changes in the amount of one or both of the conjugate variables in a point in space which arise from the Heisenberg Uncertainty Principle. Fluctuations can be classified as quantum fluctuations and thermal (classical) fluctuations. In every physical process there are always some sort of fluctuations (Mebrahtu, 2006). The level of manifestation of these fluctuations on different systems depends mainly on the system considered and also on different aspects such as temperature, the nature of particles and their interaction, and the environment of the system under consideration (Mebrahtu et al., 2006). Regardless of their level of manifestation, fluctuations do always exist intrinsically. Hence they cannot be avoided completely by any means. However it is possible to suppress or minimize them to a certain extent by different mechanisms such applying the approach of quadrature fluctuations (Mebrahtu, 2005) in a given quadrature without violating the Uncertainty Principle. Other kinds of fluctuations that exist in nature are those induced by nonlinear dynamics. Bose-Einstein condensation is characterized by a cubic nonlinearity in the so called Gross-Pitaeviskii equation. In this paper we focus on quantum fluctuations of low dimensional Bose-Einstein condensates in optical systems. 
A system which considers low dimensional ultracold atomic condensates inside a field of a laserdriven optical cavity exhibiting dispersive optical bistability at very low temperature incorporates more fluctuations due to the dispersive phenomena.

One unique feature of Bose-Einstein condensates in optical lattices is the occurrence of dynamical instability, which in a homogeneous system is only present for attractive interactions but can be induced by the presence of a periodic potential even when the interactions are repulsive. Dynamical instability implies that small deviations from the stationary solution grow exponentially in time.

Open nonlinear systems often produce bistabilities as well as dynamical phase transitions which can lead to fluctuations. A very good example of this phenomenon is the behavior of a BoseEinstein condensate (BEC) in a pumped optical cavity, where the nonlinearity is produced by the dispersive atom light interaction as indicated in the references (Brennecke et al. 2007, Colombe et al. 2007, Brennecke et al. 2008, Ritter et al. 2009, Murch et al. 2008, Gupta et al. 2007, Klinner et al. 2006, Slama et al. 2007, and Mekhoy et al. 2007). It is evident that weak cavity pumping causes classical electromagnetic fields to be built up between the mirrors of an optical cavity. In the cavity, atoms are coupled dispersively with the radiation field and detune the cavity according to the overlap of their spatial distribution with the mode function of the electric field. Then after, the cavity resonance frequency can be shifted away from or towards the frequency of the pumping laser. At this level a big variation in intensity can be induced merely by the spatial redistribution of the atoms. It is also the case that the intensity change translates into the variation of the depth of the optical dipole potential, and so it acts back upon the atomic distribution itself. In a very small region of the parameter settings close to the cavity resonance two stable or metastable configurations can exist which could give rise to a dynamical phase transition.

Non-classical states of the electromagnetic field are produced by an interaction between light and matter/atoms. At this stage, one can identify two fundamental mechanisms namely: the emission of atom radiation by resonant interaction and the interaction of light with a non-linear medium in a non-resonant process. For resonant interactions a system can be active or passive depending on the population of the resonant atomic levels. Active systems such as lasers operate with population inversion of the atomic levels.

Since recent years the atom-light interaction is one of the important issues in the study of 
ultracold quantum atomic gases. The most universal tool in accessing and studying the properties of the system of ultracold atoms is either by slowing the cloud of atoms, trapping them in classical potentials, cooling them further applying evaporative cooling which can lead to the generation of Bose-Einstein condensates, putting obstacles in their path, or finally detecting and imaging them. Besides, recent proposals have raised the possibility of quantum state preparation of the atomic ensemble with the help of measuring the output photon signal of a pumped optical resonator (Mekhov and Ritsch, 2009; Chen et al., 2007; Chen and Meystre, 2009; Marquardt and Girvin, 2009; Law, 1995; Vitali et al., 2007). The cornerstone of such a quantum state preparation with the help of a quantum nondemolition measurement is also the mutual back action between the atomic and photonic degrees of freedom. The study of correlations between atomic motion and light generated by atom-light interaction in an optical cavity is therefore of fundamental importance to our current study.

Optomechanics is an important research area on the manifestation of light-matter interaction, where the radiation pressure force of a single mode Fabry-P'erot resonator is used to manipulate the center of mass motion of a given mechanical oscillator. The reason for the popularity of optomechanical study, besides experimental realizability, is that the theoretical description could be done in a relatively simpler way, involving only few modes of the cavity field and one mode for the motion of the mirror (Genes et al., 2008; Szirmai et al., 2009). Such system is an ideal playground to test correlations between light and mesoscopic objects, to understand the underlying physics and to predict the possible applications in quantum information processing Experiments which are recently conducted with ultracold bosons in optical resonators (Mekhov and Ritsch, 2009; Chen et al., 2007; Chen and Meystre, 2009; Marquardt and Girvin, 2009) unify nicely the above concepts. Single collective motional excitations of a Bose-Einstein condensed atomic sample couples with a cavity radiation field. Starting experiments with a pure BoseEinstein condensate, other motional excitations can be safely disregarded and so a situation analogous to optomechanics can be realized without a movable mirror, but rather with the collective motion of an ensemble of atoms. The difference between the experimental tools of traditional optomechanical systems and those with ultracold gases complement each other very nicely, while the theoretical description being very similar.

The main focus of this paper is to discuss quantum fluctuations and correlations generated in a system of ultracold bosonic gases and the radiation field, particularly near a region of parameter 
settings where the system shows bistability and where the optomechanical simplification can be better manipulated. Photons leaving out of the resonator can make the cavity field noisier, which affects the dynamics of atomic motion as indicated in the reverences by Nagy et al., 2009 and Horak et al., 2000. On the other hand, quantum fluctuations of the atomic field have a back action on the photon statistics (correlations). As a result correlated fluctuations of the light and matter wave fields appear. Such fluctuations are strongly enhanced around the critical regime of bistability as it is the case with the decrease of dimensionality. Studies associated with correlations are further motivated by the need for justifying the basic assumptions of the commonly used mean-field theories as described by Horak and Ritsch, 2001; Nagy et al., 2008; Zhang et al., 2008; and Zhang et al., 2009. These authors took into account that the atom-photon cross correlations are negligible and expectation values of the light and atomic operators can be easily decoupled.

The paper is organized as follows. In the first section a general introduction is given. Section II on the other hand focuses on the model and the theoretical description of the system in the mean-field level. Other theoretical models considered in this section include cavity cooling of Bose-Einstein condensation excitations as elaborated by Horak and Ritsch, 2001, spatial selforganization of a BEC in the cavity as indicated in the works by Nagy et al., 2009; Horak et al., 2000; Horak and Ritsch, 2000; and Zhang et al., 2008, and transient collective atomic recoil lasing as verified by Zhang et al., 2008; Zhang et al., 2009; and Gopalakrishnan et al., 2009 are recapitulated here to give a full account of the mean field dynamics of a condensate in a cavity. The aim here is to arrive at the optomechanical model and verify the assumptions and approximations in order to reach at the final stage. Furthermore, a special emphasis has been given for the effect of nonlinearity, namely:

- the nonlinearity caused by atom-light interaction which is responsible for the creation of a periodic optical potential and also for an effective atom-atom collective interaction, and

- the nonlinearity caused by atomic s-wave collisions.

In section III, fluctuations around the mean field order parameter (condensate wave function) for low dimensional BEC are presented at depth and theoretical results are compared with experimental observations wherever applicable. Besides that, auto- and cross-correlations of quantum fluctuations of the fields are assessed in the stationary state formed by the balance of 
cavity loss and vacuum noise driving. Finally a summary is given in the fourth section.

\section{DESCRIPTION OF THE SYSTEM}

The system under consideration comprises of a single mode optical Fabry-Perot resonator with a waist significantly smaller than the cavity length; and a sample of low dimensional dilute ultracold bosonic atoms (Bose-Einstein condensate). Such a condensate is supposed to be a cigar-shaped along the cavity axis but with a strong transverse confinement. The radiation field inside the cavity is pumped through one of its mirrors by a laser having a frequency $\omega_{p}$ and wave number $k=\omega_{p} / c$, with $c$ being the speed of light. The laser frequency here is considered to be a far detuned from the atomic transitions. During such considerations the population of the electronic excited states is assumed to be small enough to be neglected. When the population of such exited states is negligible, the atomic internal degrees of freedom are also considered to be negligible or frozen. Consecutively the atom-light interaction is assumed to be purely dispersive. On the other hand, the cavity frequency $\omega_{c}$ for such a system has to lie close to the pump frequency $\omega_{p}$. On the other hand, the detuning frequency $\Delta_{0}=\omega_{p}-\omega_{C}$ is found to be comparable to the value of the wave number $k$. This value is, latter, considered to be half of the inverse lifetime of the photons inside the cavity.

In a frame co-rotating with the pump laser field, the total Hamiltonian of such a system can be approximately expressed as

$$
\hat{H}=\hat{H}_{A}+\hat{H}_{C}+\hat{H}_{A C}+\hat{H}_{C L}+\hat{H}_{0},
$$

where $\hat{H}_{A}$ is the Hamiltonian operator of the atoms in the ground state inside the cavity which is described as

$$
\hat{H}_{A}=\int \Psi^{+}(x)\left[\frac{-\hbar^{2}}{2 m} \frac{d^{2}}{d x^{2}}+V_{e x t}(x)+\frac{g}{2} \Psi^{+}(x) \Psi(x)\right] \Psi(x) d x,
$$

where $\Psi(x)$ is the one dimensional condensate wave function which is also referred to as the order parameter for a given condensate, $m$ is the mass of the condensed atoms, $V_{e x t}(x)$ is the external confining potential along the cavity axis and $g$ is the so-called coupling constant in one dimension. It is a characterized by the s-wave scattering constant in one-dimension. Furthermore, the term $\hat{H}_{C}$ of the Hamiltonian stands for the radiation field of the empty single- 
mode cavity which is described by

$$
\hat{H}_{C}=-\hbar \Delta_{C} \hat{a}^{+} \hat{a},
$$

where $\hbar$ is the reduced Planck's constant, $\Delta_{C}$ is the cavity coupling constant for the single-mode and $\hat{a}^{+} \hat{a}$ stands for the photon number of the radiation field or the photon number operator. The dispersive interaction between the cavity radiation field and the atoms in this low excitation limit is given by the AC-Stark shift which is also referred to as a light shift and is given by the expression:

$$
\hat{H}_{A C}=\hbar U_{0} \hat{a}^{+} \hat{a} \int \Psi^{+}(x) \Psi(x) \cos ^{2}(k x) d x,
$$

where $U_{0}$ being the single atom light-shift described by $U_{0}=g^{2} / \Delta_{A}$ which is a unique longitudinal mode and a function of the single mode cavity, i.e., $\cos (k x)$ with wave number $k=\omega_{P} / c=2 \pi / \lambda$. The Hamiltonian operator describing the coupling of the cavity field with that of the pump laser is expressibel as

$$
\hat{H}_{C L}=\eta^{*} \hat{a}+\eta \hat{a}^{+},
$$

where $\eta$ is the strength of the driving field, $\hat{a} / \hat{a}^{+}$is the annihilation/creation operators; and the asterisk on $\eta$ elaborates complex conjugation. The last part of the Hamiltonian, written as $\hat{H}_{0}=\hbar \kappa \hat{a}^{+} \hat{a}$, describes the interaction of the cavity field with the broadband reservoir of the external radiation field modes through a partially transmittive mirrors with $k$ being the cavity damping rate containing the nonlinearity $\chi^{(2)}$ (Mebrahtu, 2005). This interaction is incorporated within the Markov approximation by means of introducing a loss rate of $2 k$ and a Gaussian white noise operator $\hat{\xi}(t)$ in the Heisenberg equation of motion for the field operators as indicated by Moore et al. 2009 and the references therein.

The equation of motion or the time evaluation of the photonic annihilation operator $\hat{a}$ is given by

$$
i \frac{d}{d t} \hat{a}(t)=\left[-\Delta_{C}+\int \Psi^{+}(x, t) \Psi(x, t) U(x) d x-i k\right] \hat{a}(t)+i \hat{\eta}+i \hat{\xi}(t)
$$

with $U(x)=U_{0} \cos ^{2}(k x)$ being the local single atom light-shift, which is a periodic function with a period of $L=\lambda / 2$, since it contains the mode function squared. The operator $\hat{\xi}(t)$ describes the correlation relation: 


$$
\left\langle\hat{\xi}(t) \hat{\xi}^{+}\left(t^{\prime}\right)\right\rangle=2 k \delta\left(t-t^{\prime}\right)
$$

In Eq. (6) above, it is indicated that the dispersive interaction between the atoms and the radiation field causes a shift in the resonator frequency which is proportional to the atomic density of the condensate which can be given by $\Psi^{*}(x, t) \Psi(x, t)=|\Psi(x, t)|^{2}$. However, this frequency shift is a property of an operator and couples the equations of motion of the radiation field with those of the atomic field operators in a nonlinear way. The equation of motion of the atomic field operator on the other hand reads as

$$
i \hbar \frac{\partial}{\partial t} \Psi(x, t)=\left[-\frac{\hbar^{2} \Delta}{2 m}+V_{e x t}(x)+\hbar \hat{a}^{+}(t) \hat{a}(t) U(x)+g \Psi^{+}(x, t) \Psi(x, t)\right] \Psi(x, t) .
$$

In the atomic part of the equation of motion given above, in addition to the inert trap potential $V_{e x t}(x)$, the atom-light interaction creates a half wave-length $(\lambda / 2)$ periodic optical potential for the atoms is proportional to the dynamical photon number operator $\hat{a}^{+} \hat{a}$.

Obtaining the solutions of the coupled nonlinear operator equations described above by the equations 6, 7 and 8 simultaneously is a difficult task. Hence, to obtain the solutions of these equations; we need to apply an approximation. A more convenient approximation is found to be the usual mean-field approximation which is frequently used in determining solutions of ultracold Bose-Einstein condensates. When we separate the operators into a mean value and fluctuation part, we obtain the following expressions:

$$
\begin{aligned}
& \Psi(x, t)=\sqrt{N} \varphi(x, t)+\delta \Psi(x, t), \\
& \hat{a}(t)=\alpha(t)+\delta(t),
\end{aligned}
$$

where here $\delta \hat{\Psi}(x, t)$ represents the so-called thermal depletion or more precisely the quantum fluctuation term. The mean values are the c-numbers, defined by $\varphi(x, t)=1 / \sqrt{N}\langle\Psi(x, t)\rangle$ which, in this case is the condensate wave function. As it can be verified, the wave function is normalized to unity. The expectation value of the time dependent annihilation operator is approximated by its corresponding c-number given by $\alpha(t)=\langle\hat{a}(t)\rangle$ which is the coherent part of the cavity field. Consequently, the fluctuations have a zero expectation value. This is basically the inherent characterization of quantum fluctuations as elaborated in the reference Mebrahtu, 2006 and the references therein. The time evolution of the expectation values is 
obtained by substituting equations $9 \mathrm{a}$ and $9 \mathrm{~b}$ into the equations of motion given by equations 68 and neglecting all terms containing fluctuations. By carrying out such an approximation one can obtain the expression for the condensate which is referred to as the Gross-Pitaevskii equation (GPE):

$$
\begin{gathered}
i \frac{d}{d t} \hat{a}(t)=\left[-\Delta_{C}+N\langle U\rangle-i k\right] \hat{a}(t)+i \hat{\eta}, \\
i \hbar \frac{\partial}{\partial t} \varphi(x, t)=\left[-\frac{\hbar^{2} \Delta}{2 m}+V_{e x t}(x)+\hbar|\alpha(t)|^{2} U(x)+g N|\varphi(x, t)|^{2}\right] \varphi(x, t)
\end{gathered}
$$

where the expectation value $\langle U\rangle=\int \varphi^{*}(x, t) U(x) \varphi(x, t) d x$. The time evolution of equation 10a is governed by two characteristic frequencies, namely the detuning $\Delta_{0}$ and the photon loss rate $k$. Equation 10b, on the other hand, indicates that the characteristic frequency is set by the recoil frequency $\omega_{R}=\hbar k^{2} / 2 m$. In this situation the dynamics of the resonator field relaxes very fast in comparison with the dynamics of the atomic motion; and therefore can be considered instantaneous with respect to the relaxation of the condensate. At this stage, we can also assume that for any given atomic configuration the resonator field has already reached its steady state value, which, according to Eq. (10a), is given by

$$
\alpha_{S S}=\frac{i \eta}{\Delta_{0}-N\langle U\rangle+i k} .
$$

Hence, at the end, one needs only to integrate Eq. (10b) in order to adiabatically eliminate $\alpha(t)$. It is worth noting that if the time scales of the resonator field and the condensate dynamics do not differ that much, some complex coupled solutions can exist, which need a simultaneous integration of equations 10a and 10b.

A transformation of a thermodynamic system can be considered adiabatic when it is quick enough so that no significant heat transfer happens between the system and the outside environment. On the contrary, a transformation of a thermodynamic system can be considered isothermal if it is slow enough so that the system's temperature can be maintained during a heat exchange with the outside environment (Mebrahtu, 2006 and references therein).

After the adiabatic elimination of the photon field, the most direct method to calculate the steady state of the condensate wave function $\varphi(x)$ is the one based on the imaginary-time propagation based on equation 10b given above. In real-time, the steady-state solution has the time 
dependence:

$$
\varphi(x, t)=\varphi(x) e^{-i \mu t / \hbar}
$$

where $\mu / \hbar$ is the lowest frequency eigenvalue of the nonlinear problem of equation 10b. In the imaginary time, all fluctuations around the steady-state die out, since they propagate with higher frequencies in real time and consequently vanish faster in imaginary time than the steady-state solution. In such a situation, one just needs to renormalize the solution $\varphi(x, t)$ after some finite propagation time. Note also that, since all the quantities on the left hand side of equation 10b are real, the condensate wave function $\varphi(x)$ can also be chosen to be real. We also note that, , as we will see it later, in the case of an effective blue detuning given by $\Delta_{0}-N\langle U\rangle>0$, the resonator field may heat the atomic motion since some excitations might have positive imaginary parts, and there is no steady-state condensate wave function at all. However, due to the method of imaginary time propagation, one can find a condensate wave function, even in this case, corresponding to a dynamically unstable equilibrium situation.

\section{FLUCTUATIONS AROUND THE MEAN FIELD ORDER PARAMETER}

For low dimensional Bose-Einstein condensates quantum fluctuations are expressed in the form of density and phase fluctuations. In the mean field regime of a condensate, a huge interest has been devoted to the study of fluctuations of non-linear dynamics of condensed systems. Having obtained the steady-state values of the low dimensional Bose-Einstein condensate wave functions and the resonator field amplitudes in a cavity, one can look for the fluctuations of the annihilation (creation) operators described as $\delta \hat{a}(t), \delta \Psi(x, t) \quad\left(\delta \hat{a}^{+}(t), \delta \Psi^{+}(x, t)\right)$ respectively in a linear order. This linear stability analysis corresponds to the famous Bogoliubov theory of low dimensional condensates as described in the references described by Fallani et al. 2005, Louisell, 1973, and Castin, 2001. This approach has an analogy to the optomechanics, and other nonlinear systems, especially in hydrodynamics. Inserting the separation of the field operators of equation

9 into equations 6 and 8, and neglecting fluctuations higher than the first order, we can easily arrive at the following equations:

$$
i \frac{d}{d t} \delta \hat{a}(t)=\left[-\Delta_{C}+N\langle U\rangle-i k\right] \delta \hat{a}(t)+N \alpha_{S S} \int \varphi(x) U(x)\left[\delta \hat{\Psi}(x, t)+\delta \hat{\Psi}^{+}(x, t)\right] d x+i \xi(t),
$$




$$
\begin{aligned}
& i \hbar \frac{\partial}{\partial t} \delta \hat{\Psi}(x, t)=\left[-\frac{\hbar^{2} \Delta}{2 m}+V_{e x t}(x)+\hbar\left|\alpha_{S S}\right|^{2} U(x)-\mu+g N|\varphi(x)|^{2}\right] \delta \hat{\Psi}(x, t)+ \\
& \hbar U(x) \varphi(x)\left[\alpha_{S S}^{*} \delta \hat{a}(t)+\alpha_{S S} \delta \hat{a}^{+}(t)\right]+g N|\varphi(x)|^{2}\left[\delta \hat{\Psi}(x, t)+\delta \hat{\Psi}^{+}(x, t)\right]
\end{aligned}
$$

where the zeroth order terms have been canceled, since they fulfill Eq. (10b) and (11) with $d \alpha_{S S} / d t=0$. Here the fluctuation term which is a combination of low dimensionality of the condensate and the effect of the condensate being in a cavity which has dispersive phenomena is given by the expression $\delta \hat{\Psi}(x, t)=N^{-1 / 2} \delta \Psi(x, t) e^{i \mu t / \hbar}$ has been introduced.

A closer look into equation 13 reveals that the time evolution of the annihilation operators coupled to those of the creation operators. It is a consequence of the complex nature of the photonic and particle fields. In order to diagonalize equation 13, it is possible to choose two equivalent ways:

- First one can separate the complex quantities into real and imaginary parts and study their time evolution; this is the approach mainly used in optomechanical studies.

- The other way is to diagonalize the set of equations not only just containing $\delta \hat{a}$ and $\delta \hat{\Psi}$, but also their Hermitian adjoints $\delta \hat{a}^{+}$and $\delta \hat{\Psi}^{+}$; this approach is familiar with the Bogoliubov-de Gennes theory of superfluidity.

\section{CONCLUSION}

In this paper we have studied the dynamics, effects of interactions and the manifestation of quantum fluctuations of low dimensional Bose-Einstein condensate inside an optical cavity. As the dispersive atom-photon interaction couples with the atomic motion and the dynamics of the photonic field in a nonlinear way, strong correlations are expected to show up. These interactions not only cause cavity cooling or cavity heating but also alter the statistics of the constituent subsystems. The strength of the coupling is verified to be inversely proportional to the detuning of the pump photon frequency. Such a system which considers low dimensional ultracold atomic condensates inside a field of a laser-driven optical cavity exhibiting dispersive optical bistability at very low temperature shows enhanced fluctuations due to the dispersive phenomena.

We have analyzed that the mapping of the original system to a two mode effective model in which only the two highest populated one-particle states are kept from a plane-wave expansion of the atomic motion. Furthermore, we have compared the mean-field solution and the 
fluctuation spectrum of the optomechanical model to that of the model which is not restricted to the first two highly occupied modes. In the cavity heating region, the unstable polariton mode can have a positive imaginary part in the order of a kilohertz giving an evaporation rate of the Bose-Einstein condensate in milliseconds. It is evident that such a timescale is in the experimental reach.

The experimental progress in combining cavity quantum electrodynamics systems with ultracold atoms promises an interesting playground to test the manifestation of light-matter interactions in the mesoscopic scale. In such systems both the radiation and the atomic part are dynamical entities. The better understanding of their interplay can have a significant impact not just on our knowledge of nonequilibrium systems, but also on the implementations of quantum information processing devices or quantum simulators of other systems.

It is evident that low dimensional quantum gases or ultracold gases exhibit fascinating properties and attracted a lot of attention both theoretically and experimentally. However it should be also stressed that, when viewed theoretically, the use of a mean field theory to describe quantum gases in low dimensional Bose gases is highly restricted to extremely low temperatures near the absolute zero. The possibility of generating low dimensional bosonic gases (condensates) being possible in principle is characterized by the manifestation of intrinsic quantum fluctuations which may result in destroying the very existence of the condensate at low dimension.

\section{ACKNOWLEDGEMENTS}

I would like to acknowledge and thank to my university, the Mekelle University, my college: the College of Natural and Computational Sciences and my department: the Department of Physics for their support during this work.

\section{REFERENCES}

Brennecke, F., Donner, T., Ritter, S., Bourdel, T., Köhl, M \& Esslinger, T. 2007. Cavity QE with a Bose-Einstein condensate. Nature, 450: 268-271.

Brennecke, F., Ritter, S., Donner, T \& Esslinger, T. 2008. Cavity Optomechanics with a Bose Einstein Condensate. Science, 322: 235-238.

Castin, Y. 2001. Coherent atomic matter waves. In: R. Kaiser, C. Westbrook, \& F. David (Eds), Lecture Notes of Les Houches Summer School, EDP Sciences and Springer-Verlag. 
Chen, W \& Meystre, P. 2009. Cavity QED characterization of many-body atomic states in double-well potentials: Role of dissipation. Phys. Rev. A., 79: 043801-043808.

Chen, W., Meiser, D \& Meystre, P. 2007. Cavity QED determination of atomic number statistics in optical lattices, Phys. Rev. A., 75: 023812-023820.

Colombe, Y., Steinmetz, T., Dubois, G., Linke, F., Hunger, D, \& Reichel, J. 2007. Strong atomfield coupling for Bose-Einstein condensates in an optical cavity on a chip. Nature, 450: 272-275.

Fallani, L., Fort, C., Piovella, N., Cola, M., Cataliotti, F. S., Inguscio, M \& Bonifacio, R. 2005. Collective atomic recoil in a moving Bose-Einstein condensate: From Superradiance to Bragg scattering. Phys. Rev. A., 71: 033612-033619.

Genes, C., Mari, A., Tombesi, P., \& Vitali, D. 2008. Robust entanglement of a micromechanical resonator with output optical fields. Phys. Rev. A., 78: 032316-032329.

Gopalakrishnan, S., Lev, B. L., \& Goldbart, P. M. 2009. Emergent crystallinity and frustration with Bose-Einstein condensates in multimode cavities. Nature Physics, 5: 845-850.

Gupta, S., Moore, K. L., Murch, K. W \& Stamper, Kurn D. M. 2007. Cavity Nonlinear Optics at Low Photon Numbers from Collective Atomic Motion. Phys. Rev. Lett., 99: 213601213605.

Horak, P. \& Ritsch, H. 2001. Dissipative dynamics of Bose condensates in optical cavities. Phys. Rev. A., 63: 023603-0233611.

Horak, P., Barnett, S. M., \& Ritsch, H. 2000. Coherent dynamics of Bose-Einstein condensates in high-finesse optical cavities. Phys. Rev. A., 61: 033609-033613.

Klinner, J., Lindholdt, M., Nagorny, B., \& Hemmerich, A. 2006. Normal Mode Splitting and Mechanical Effects of an Optical Lattice in a Ring Cavity. Phys. Rev. Lett., 96: 023002023005.

Law, C. K. 1995. Interaction between a moving mirror and radiation pressure: A Hamiltonian formulation. Phys. Rev. A., 51: 2537-2541.

Louisell, W. H. 1973. Quantum statistical properties of radiation. John Wiley \& Sons, New York.

Marquardt, F. \& Girvin, S. M. 2009. Optomechaics. Physics, 2: 40-46.

Marquardt, F., Harris, J. G. E \& Girvin S. M. 2006. Dynamical Multistability Induced by Radiation Pressure in High-Finesse Micromechanical Optical Cavities. Physical Review 
Letters, 96: 103901-103904.

Mebrahtu, A. 2006. Fluctuations in Quantum Optical Systems: From Bose-Einstein Condensates to Squeezed States of Light (Der Andere Verlag, ISBN-10: 3899595602, Berlin, 2006).

Mebrahtu, A. 2005. Parametric oscillation with squeezed vacuum reservoirs. J. Mod. Opt., 52: 813-832

Mebrahtu, A., Sanppera, A \& Lewenstein, M. 2006. Splitting and merging an elongated BoseEinstein condensate at finite temperature. Phys. Rev. A., 73: 033601-033608

Mekhov, I. B \& Ritsch, H. 2009. Quantum optics with quantum gases: Controlled state reduction by designed light scattering. Phys. Rev. A., 80: 013604-013604.

Mekhov, I. B. \& Ritsch, H. 2009. Quantum Nondemolition Measurements and State Preparation in Quantum Gases by Light Detection. Phys. Rev. Lett., 102: 020403-020407.

Mekhov, I.B., Maschler, C \& Ritsch, H. 2007. Cavity-enhanced light scattering in optical lattices to probe atomic quantum statistics. Phys. Rev. Lett., 98: 100402- 100405.

Moore, M. G., Zobay, O \& Meystre, P. 1999. Quantum optics of a Bose-Einstein condensate coupled to a quantized light field. Phys. Rev. A., 60: $1491-1506$.

Murch, K. W., Moore, K. L., Gupta, S \& Stamper-Kurn, D. M. 2008. Observation of quantum measurement back action with an ultracold atomic gas. Nature Physics, 4: 561-564.

Nagy, D., Domokos, P., Vukics, A \& Ritsch, H. 2009. Nonlinear quantum dynamics of two BEC modes dispersively coupled by an optical cavity. Eur. Phys. J. D, 55: 659-668.

Nagy, D., Szirmai G \& Domokos, P. 2008. Self-organization of a Bose-Einstein condensate in and optical cavity. Eur., Phys. J. D, 48: 127-137.

Ritter, S., Brennecke, F., Baumann, K., Donner, T., Guerlin, C \& Esslinger, T. 2009. Dynamical Coupling between a Bose-Einstein Condensate and a Cavity Optical Lattice. Appl. Phys. B., 95: 213-218.

Slama, S., Krenz, G., Bux, S., Zimmermann, C \& Courteille, P. W. 2007. Cavity-enhanced superradiant Rayleigh scattering with ultracold and Bose-Einstein condensed atoms. Phys. Rev. A., 75: 063620-063629.

Szirmai, G., Nagy, D \& Domokos, P. 2009. Excess Noise Depletion of a Bose-Einstein Condensate in an Optical Cavity. Phys. Rev. Lett., 102: 080401-080404.

Vitali, D., Gigan, S., Ferreira, A., Böhm, H. R., Tombesi, P., Guerreiro, A., Vedral, V., Zeilinger, A \& Aspelmeyer, M. 2007. Optomechanical Entanglement between a Movable Mirror 
and a Cavity Field. Phys. Rev. Lett., 98: 030405-030408.

Zhang, J. M., Cui, F. C., Zhou, D. L \& Liu, W. M. 2009. Nonlinear dynamics of a cigar-shaped Bose-Einstein condensate in an optical cavity Phys. Rev. A., 79: 033401-033408.

Zhang, J. M., Liu, W. M \& Zhou, D. L. 2008. Cavity QED with cold atoms trapped in a doublewell potential. Phys. Rev. A., 77: 033620-033627. 